\title{
A RARE CASE REPORT OF ACALCULOUS CHOLECYSTITIS IN A DENGUE ATTACKED PREGNANT WOMAN
}

\author{
K. Ravichandran ${ }^{1}$, M. Senthil Velan², M. Karthik³, Imran Thariq Ajmal ${ }^{4}$, U. Srinivasulu ${ }^{5}$
}

\section{HOW TO CITE THIS ARTICLE:}

K. Ravichandran, M. Senthil Velan, M. Karthik, Imran Thariq Ajmal, U. Srinivasulu. "A Rare Case Report of Acalculous Cholecystitis in a Dengue Attacked Pregnant Woman". Journal of Evolution of Medical and Dental Sciences 2015; Vol. 4, Issue 05, January 15; Page: 884-887, DOI: 10.14260/jemds/2015/126

\begin{abstract}
Dengue viral infections present a significant risk during pregnancy to both mother and fetus. A young woman at 13 weeks' gestation presented with fever and abdominal pain. Over the course of 5 days, she developed nausea, petechiae, severe thrombocytopenia, and acalculous cholecystitis. After a serologic diagnosis of acute infection with Dengue virus, she was provided supportive care. An uncomplicated pregnancy led to delivery of a healthy infant at 40 weeks gestation. Pregnancies complicated by dengue infection require close monitoring for potential maternal and fetal complications.
\end{abstract}

KEYWORDS: dengue fever, pregnancy, acalculous cholecystitis, thrombocytopenia.

INTRODUCTION: Dengue fever is a mosquito-borne flaviviral infection endemic in the tropics and subtropics affecting up to 100 million people per year.[1] The presentation of Dengue fever can be protean; infection with the dengue virus can be either asymptomatic, or can present solely as fever. A subset of patients can progress to involve hemorrhagic manifestations such as ecchymoses, gastrointestinal bleeding, or epitstaxis. When hemorrhagic symptoms occur with plasma leakage (Ascites or pleural effusions) and shock, the most lethal form of the disease, dengue hemorrhagic fever can occur.[1] Four distinct dengue viral serotypes (DEN 1-4) are known to cause illness. Infection with one serotype confers protection to re-infection with the same serotype, while reinfection with dissimilar serotypes confers no long-term protection and may even predispose to worse clinical outcome.

We describe the clinical and laboratory course of a young woman infected with Dengue during the first trimester.

CASE: A 29 year old Gravida 1 Para 0 woman presented at 13 weeks' gestational age to the emergency room at our centre fever and abdominal pain with chills, severe myalgia and arthralgia. Past medical, surgical, family history and review of systems were otherwise unremarkable. She was married, worked as a nurse and did not have a history of drug, tobacco or alcohol abuse.

An evaluation in the emergency room included blood cultures, labs, and lower extremity doppler venous duplex ultrasound for her leg pain. These tests showed no evidence of bacteremia, leukocytosis, or deep venous thrombosis, and the platelet count was 120,000 per microliter (normal range 150,000-450,000). She was treated with intravenous hydration and sent home. Three days later she re-presented with right upper quadrant abdominal pain, and severe thrombocytopenia (17,000 platelets per microliter). Magnetic resonance imaging and abdominal ultrasound revealed significant gall bladder wall thickening, pericholecystic fluid collection, an ultrasonographic Murphy's sign, but no cholelithiasis, consistent with the diagnosis of acalculous cholecystitis. Obstetric ultrasound documented fetal viability with a crown rump length consistent with 13 weeks' gestation 


\section{CASE REPORT}

and a heart rate of 155 beats per minute. Physical examination was unremarkable except for right upper quadrant tenderness with guarding and multiple small petechiae over her bilateral upper extremities. Blood smear at that time showed no schistocytes or abnormal red blood cells (RBCs). The patient was transferred to a tertiary care center where repeat laboratory evaluation was remarkable for a platelet count of 14,000 per microliter, white blood cell (WBC) count of 4.4 units with immature granulocytes and bands present, aspartate transaminase (AST) of $319 \mathrm{U} / \mathrm{L}$, alanine transaminase (ALT) of $198 \mathrm{U} / \mathrm{L}$, lipase of $22 \mathrm{U} / \mathrm{L}$, alkaline phosphatase of $74 \mathrm{U} / \mathrm{L}$, creatinine of $0.5 \mathrm{mg} / \mathrm{dl}$, fibrinogen of $302 \mathrm{mg} / \mathrm{dl}$, and total bilirubin of $0.8 \mathrm{mg} / \mathrm{dl}$. Her stool examination showed no ova or parasites, staining for Cryptosporidium, Cyclospora, and Isospora were all negative, and cultures did not grow E. coli, Y. Enterocolitica, Salmonella spp., Shigella spp., Aeromonas spp., Plesiomonas spp., or Vibrio spp Antibodies to hepatitis A virus (HAV) IgM and IgG were negative, hepatitis B virus (HBV) surface antigen and core antigen was negative with a positive HBV surface antibody, and hepatitis $C$ virus (HCV) antibody was negative. Blood cultures and thick and thin smear were negative for Plasmodium falciparum malaria.

The patient was started on antibiotic therapy (ampicillin, gentamicin, and azithromycin) and admitted to the hospital. General surgical consultation was obtained for possible cholecystectomy, but conservative management with hospital monitoring, platelet transfusion, and antibiotic therapy was recommended. Ten days after onset of symptoms, acute serologies by enzyme-linked immunosorbant assay for Dengue virus revealed titers of IgG and IgM at 13.58 and 10.50 Index Value respectively (Negative < .90 Index Value, equivocal 0.9 - 1.10 Index Value), suggestive of secondary re-infection with dissimilar serotype or acute primary infection undergoing seroconversion. ${ }^{[2]}$ While in the hospital she was afebrile. Platelets and liver transaminases returned to normal after five days of antibiotic administration and observation. She finished a course of intravenous ampicillin and gentamicin before the results of the dengue virus serology were known. The remainder of the pregnancy was uneventful. Ultrasound fetal assessment showed normal fetal growth. The patient had an uncomplicated vaginal delivery at 40 and $1 / 7$ weeks gestation after spontaneous labor. She delivered a healthy, live born female infant, APGARS 9 and 10, weight 3436 G. There were no gross abnormalities of the placenta.

DISCUSSION: The differential of fever, abdominal pain, and thrombocytopenia in a pregnant patient returning from the tropics is lengthy, and must include malaria, acute gastroenteritis, typhoid fever, and dengue fever. Any bacterium (such as E. coli, Y. Enterocolitica, Salmonella spp., Shigella spp., Aeromonas spp., Plesiomonas spp., and Vibrio spp) with propensity to transverse the gut mucosa and enter the bloodstream could potentially cause sepsis and therefore thrombocytopenia in returning travelers. The relative stability of the patient's vital signs, indolent clinical course, and absence of evidence for disseminated intravascular coagulation made bacterial sepsis unlikely. Typhoid fever, caused by Salmonella enteritidis subtype typhi or paratyphi, would be another consideration, especially in a patient with radiographic cholecystitis, fever, and abnormal liver function tests, but negative blood cultures, normal vital signs, and normal erythrocyte sedimentation rate (ESR) of 9 $\mathrm{mm} / \mathrm{hr}$ was evidence against bacterial infection.

The acute syndrome of severe myalgias, fever, followed by abdominal pain and thrombocytopenia in the patient above is typical for Dengue fever. Dengue fever is caused by a mosquito-transmitted flavivirus that is endemic throughout the Caribbean and Mexico, and much of 


\section{CASE REPORT}

the tropical world. There are four distinct viral serotypes (DEN 1-4) that can cause clinical symptoms. [3] Symptoms arise 4-7 days after the bite of a mosquito, but can be as short as 3 days or a long as 14 days following insect exposure. The clinical syndrome of classic dengue fever is characterized initially by headache, retro-orbital eye pain, and severe myalgias and arthalgias originally termed "break bone" fever. Rash, gastrointestinal pain, and diarrhea are common manifestations of the syndrome. The fever typically lasts for 5-7 days and may have a biphasic appearance in some. Upon resolution of the fever, patients often run the risk of hemorrhagic symptoms (Epistaxis, ecchymoses, and gastrointestinal bleeding) and plasma leakage syndrome (Hemoconcentration, ascites, or pleural effusions) called dengue hemorrhagic fever[4,5]. Dengue hemorrhagic fever may have a mortality of $5 \%$ in those untreated, but this may be lowered to $1 \%$ with effective resuscitation with intravenous fluids.[6] Our patient above had no evidence of hemorrhagic manifestations or plasma leakage syndrome consistent with Dengue hemorrhagic fever.

Little is known about the effect of the Dengue viral infection in pregnancy on fetal and maternal outcome. The effect of the virus on the developing fetus in the first and second trimester is controversial. A prospective case series in French Guiana of 22 women with confirmed or probable Dengue syndrome suggested a trend towards increased fetal demise $(13.6 \%$ in fetuses of mothers infected with dengue vs. $1.9 \%$ in other hospitalized mothers, $p=0.2$ ). ${ }^{77]}$ There was no increase in premature deliveries related to dengue infection (13.6\% vs. $11 \%$ in hospital controls). No fetal malformations were identified in this study. Another retrospective Vietnamese study from the 1950s also did not find significant differences in fetal death, premature deliveries, or malformations, but failed to take into consideration infections with malaria.[1]

Our patient presented with acalculous cholecystitis, a rare but described complication of Dengue infection. The optimal treatment and prognosis of patients with acalculous cholecystitis due to Dengue fever was examined retrospectively in a series of 131 patients with Dengue fever in Taiwan. ${ }^{[2]}$ In this series about $8 \%$ of the patients had clinical and radiographic evidence of acalculous cholecystitis as defined by gall bladder wall thickening, pericholecystic fluid and positive Murphy's sign. In this series of those who had cholecystitis, both thrombocytopenia and transaminitis were uniformly evident (100\%), but obstructive cholangiopathy was only evident in $50 \%$ of the patients.

All of the patients who underwent a therapeutic procedure (cholecystectomy or percutaneous cholecystotomy) had problems with post-procedural bleeding requiring transfusion and prolonged hospital stays ( $4.1 \pm 1.6$ days for conservative therapy vs. 18 and 16 days for those requiring invasive therapy). Consequently, many recommend conservative management if diffuse peritonitis or perforation is not suspected.

In the absence of effective therapies for Dengue viral infections, and given the evidence to date for an increased risk of complicated pregnancies, prevention of infection remains the mainstay of treatment. Prophylaxis with anti-mosquito netting and insect repellant is recommended for travelers to endemic areas. ${ }^{[3]}$ According to animal and human studies the insect repellant DEET (N,Ndiethyl-m-toluamide) is safe during pregnancy.[8,9] Use of insecticide-treated nets during pregnancy reduces malaria-related fetal loss without significant harm to the fetus.[10]

A diagnosis of Dengue should be entertained in persons with persistent fevers, diarrhea, abdominal pain or thrombocytopenia. Future studies are needed to determine more precise estimates of the morbidity and mortality of this infection in gravid women and their fetuses and optimal management strategies. 


\section{REFERENCES:}

1. Mirovsky J, Holub J, Nguyen Ba C. Influence of dengue on pregnancy and the fetus. Gynecol Obstet (Paris) 1965; 64: 673-6. [PubMed]

2. Wu KL, Changchien CS, Kuo CM, Chuah SK, Lu SN, Eng HL, et al. Dengue fever with acute acalculous cholecystitis. Am J Trop Med Hyg. 2003; 68: 657-60. [PubMed]

3. Wilder-Smith A, Schwartz E. Dengue in travelers. N Engl J Med. 2005; 353: 924-32. [PubMed]

4. Kao CL, King CC, Chao DY, Wu HL, Chang GJ. Laboratory diagnosis of dengue virus infection: current and future perspectives in clinical diagnosis and public health. J Microbiol Immunol Infect. 2005; 38: 5-16. [PubMed].

5. Miranda Franco R, Casta Velez A. Eradication of malaria in Puerto Rico. Rev Panam Salud Publica. 1997; 2: 146-50. [PubMed]

6. Wills BA, Nguyen MD, Ha TL, Dong TH, Tran TN, Le TT, et al. Comparison of three fluid solutions for resuscitation in dengue shock syndrome. N Engl J Med. 2005; 353: 877-89. [PubMed]

7. Carles G, Peiffer H, Talarmin A. Effects of dengue fever during pregnancy in French Guiana. Clin Infect Dis. 1999; 28: 637-40. [PubMed]

8. McGready R, Hamilton KA, Simpson JA, Cho T, Luxemburger C, Edwards R, et al. Safety of the insect repellent N,N-diethyl-M-toluamide (DEET) in pregnancy. Am J Trop Med Hyg. 2001; 65: 285-9. [PubMed]

9. Schoenig GP, Neeper-Bradley TL, Fisher LC, Hartnagel RE., Jr Teratologic evaluations of N,Ndiethyl-m-toluamide (DEET) in rats and rabbits. Fundam Appl Toxicol. 1994; 23: 63-9. [PubMed].

10. Gamble C, Ekwaru JP, ter Kuile FO. Insecticide-treated nets for preventing malaria in pregnancy. Cochrane database of systematic reviews (Online) 2006: CD003755. [PubMed]

\section{AUTHORS:}

1. K. Ravichandran

2. M. Senthil Velan

3. M. Karthik

4. Imran Thariq Ajmal

5. U. Srinivasulu

PARTICULARS OF CONTRIBUTORS:

1. Assistant Professor, Department of General Surgery, Rajah Muthaiah Medical College.

2. Professor, Department of General Surgery, Rajah Muthaiah Medical College.

3. Assistant Professor, Department of General Surgery, Rajah Muthaiah Medical College.
4. Assistant Professor, Department of General Surgery, Rajah Muthaiah Medical College.

5. Final Year Post Graduate Student, Department of General Surgery, Rajah Muthaiah Medical College.

\section{NAME ADDRESS EMAIL ID OF THE CORRESPONDING AUTHOR:}

Dr. K. Ravichandran, \# 21, Venugopal Street, Chidambaram-608001.

E-mail: drkravichandranyadav@gmail.com

Date of Submission: 09/12/2014.

Date of Peer Review: 10/12/2014.

Date of Acceptance: 22/12/2014.

Date of Publishing: 14/01/2015. 